University of Nebraska - Lincoln

DigitalCommons@University of Nebraska - Lincoln

$2-2021$

\title{
Interpretation of ambiguous pronouns in adults with intellectual disabilities
}

Kara Hawthorne

Susan J. Loveall

Follow this and additional works at: https://digitalcommons.unl.edu/specedfacpub

Part of the Special Education and Teaching Commons

This Article is brought to you for free and open access by the Department of Special Education and Communication Disorders at DigitalCommons@University of Nebraska - Lincoln. It has been accepted for inclusion in Special Education and Communication Disorders Faculty Publications by an authorized administrator of DigitalCommons@University of Nebraska - Lincoln. 


\title{
Interpretation of ambiguous pronouns in adults with intellectual disabilities
}

\author{
Kara Hawthorne \\ Department of Hearing, Speech, and Language Sciences, Sorenson Language and Commu- \\ nication Center, Gallaudet University, Washington, DC, USA \\ Susan J. Loveall \\ Department of Special Education and Communication Disorders, \\ University of Nebraska-Lincoln, Lincoln, NE, USA

\begin{abstract}
Correspondence - Assistant Professor Kara Hawthorne, Department of Hearing, Speech, and Language Sciences, Sorenson Language and Communication Center, Gallaudet University,
\end{abstract} \\ Washington, DC 20002, USA; email kara.hawthorne@gallaudet.edu
}

\begin{abstract}
Background - Pronouns are referentially ambiguous (e.g. she could refer to any female), yet they are common in everyday conversations. Individuals with typical development (TD) employ several strategies to avoid pronoun interpretation errors, including the subject bias - an assumption that a pronoun typically refers to the subject (or, with the closely related order-of-mention bias, the first-mentioned character) of the previous sentence. However, it is unknown if adults with intellectual disability (ID) share this strategy or the extent to which the subject bias is associated with non-verbal abilities or receptive vocabulary. Methods - We tested 22 adults with mixed-aetiology ID on their interpretation of ambiguous pronouns using the visual world eye-tracking paradigm and by asking a follow-up pronoun interpretation question. A group of TD adults was also
\end{abstract}

Published in Journal of Intellectual Disability Research, vol 65, part 12 (February 2021), pp 125-132.

doi: $10.1111 /$ jir.12801

Copyright (c) 2020 MENCAP and International Association of the Scientific Study of Intellectual and Developmental Disabilities and John Wiley \& Sons Ltd. Used by permission.

Accepted 16 November 2020. 
tested to establish the strength of the subject bias with our materials and task. Results - Adults with ID did demonstrate the subject bias, but it was significantly less robust than that seen in TD. For participants with ID, the subject bias was influenced by non-verbal IQ and receptive vocabulary at different stages of processing.

Conclusions - Given the frequency of pronouns in conversation, strengthening the subject bias may help alleviate discourse and reading comprehension challenges for individuals with ID, particularly those with lower non-verbal and/or vocabulary skills.

Keywords: discourse comprehension, mixed-aetiology intellectual disability, pronouns, referential ambiguity, subject bias

\section{Introduction}

Pronouns are common in conversation but can be difficult to interpret because they are referentially ambiguous (e.g. she could refer to any female). Because pronouns establish meaning at both the sentence and discourse levels, errors in linking pronouns with their intended referents can cause significant confusion. Individuals with typical development (TD) integrate several sources of information when identifying a pronoun's referent, for example, animacy, gender and number information encoded in the pronoun itself, as well as information from the broader discourse/conversational context (e.g. Arnold et al., 2000; Song and Fischer 2005; Tyler and Marslen-Wilson 1982).

One well-studied pronoun interpretation strategy is the subject bias: an assumption that a pronoun typically refers to the subject of the previous sentence. For example, in 'Kara texted Susan. She wanted ... ', listeners assume she refers to Kara (Frederiksen 1981). A related strategy is the order-of-mention bias, which is the assumption that a pronoun refers to the character that was mentioned first in the previous sentence (Gernsbacher and Hargreaves 1998). These two biases have been frequently replicated in adults (Järvikivi et al. 2005), and similar effects have been observed in 2.5- to 5-year-old TD children (Song and Fisher, 2007; Hartshorne et al. 2015). In English, the subject and order-of-mention biases usually lead to the same conclusion because subjects are typically mentioned before objects (although there are low-frequency sentence types that are exceptions, such as the passive 'Susan was texted by Kara' or object-clefted 'It was Susan who Kara texted'). For this paper, we used the common 
subject-verb-object pattern of English, so we do not distinguish between the subject bias and order-of-mention bias. We will use the term 'subject bias' throughout for conciseness.

Very little is known about pronoun interpretation in individuals with intellectual disability (ID) (although see Perovic 2006; Perovic et al. 2013; Tavares et al. 2015). Establishing whether this population has a subject bias is an important precursor to investigating other cues that may also influence pronoun interpretation, as research in TD suggests that other cues interact with the subject bias (e.g. Hawthorne et al. 2016). Therefore, our first aim was to determine if adults with ID show the subject bias or, alternatively, if they show either an object bias (because objects typically occur closer to the pronoun) or no preference for subject versus object antecedents. Our second aim was to examine the relation between the subject bias and non-verbal and verbal abilities in this population.

\section{Materials and methods}

\section{Participants}

Participants were 22 adults with mixed-aetiology ID recruited from an intermediate care facility, which provided diagnostic information. In addition to participants with a primary diagnosis of ID $(n=12)$, several also had co-morbid diagnoses: Prader-Willi syndrome ( $n=$ $3)$, autism spectrum disorder $(n=2)$, cerebral palsy $(n=2)$, autism spectrum disorder and cerebral palsy $(n=1)$, Lesch Nyhan syndrome $(n=1)$ and fetal alcohol syndrome $(n=1)$. Participants with ID were included if they had an IQ $\leq 70$ and if the facility reported no dementia or age-related cognitive declines and language abilities sufficient to complete the tasks (commensurate with $\geq 3$ years). Participants were excluded if they failed to meet the 70\% cut-off criterion for control trials (see Control trials and animal check; $n=6$ excluded from an original $n=28$ ).

An additional sample of 27 adult college students served as a model of the subject bias in typical adults and to establish the strength of the subject bias using our stimuli and tasks. TD participants were included if their IQ fell within one standard deviation of the normative mean (i.e. 85-115). 
Table 1 Participant characteristics.

\begin{tabular}{lll} 
Group & $I D$ & $T D$ \\
\hline n (female:male) & $22(8: 14)$ & $27(21: 6)$ \\
Age & $\mathrm{M}=48, \mathrm{Md}=42, \mathrm{SD}=14$ & $\mathrm{M}=21, \mathrm{Md}=21, \mathrm{SD}=1$ \\
IQ: KBIT-2 SS & $\mathrm{M}=49, \mathrm{Md}=48, \mathrm{SD}=7$ & $\mathrm{M}=100, \mathrm{Md}=103, \mathrm{SD}=9$ \\
Non-verbal IQ: KBIT-2 non-verbal SS & $\mathrm{M}=50, \mathrm{Md}=5 \mathrm{O}, \mathrm{SD}=9$ & $\mathrm{M}=99, \mathrm{Md}=98, \mathrm{SD}=10$ \\
Receptive Vocabulary: PPVT-4 GSV & $\mathrm{M}=15 \mathrm{O}, \mathrm{Md}=148 \mathrm{SD}=28$ & $\mathrm{M}=219, \mathrm{Md}=219, \mathrm{SD}=7$
\end{tabular}

ID, intellectual disability; TD, typical development

$\mathrm{M}$, mean; Md, median; SD, standard deviation

SS, standard scores; GSV, growth score values

KBIT-2, Kaufman Brief Intelligence Test - 2nd Edition

PPVT-4, Peabody Picture Vocabulary Test - 4th Edition

All participants were native English speakers and had adequate hearing and vision to complete the tasks, as assessed during a pre-experiment screening. Participant characteristics are reported in Table 1.

This study received research ethics approval from the Institutional Review Board at the University of Mississippi. Informed consent was obtained from participants with TD and from parents/legal guardians of participants with ID. Participants with ID gave verbal assent before testing, after the researcher reviewed the study objectives and tasks and gave an opportunity to ask questions.

\section{Assessment of IQ and receptive vocabulary}

IQ and non-verbal ability were assessed using the Kaufman Brief Intelligence Test - 2nd Edition (KBIT-2; Kaufman and Kaufman, 2004), and receptive vocabulary was assessed with the Peabody Picture Vocabulary Test - 4th Edition (PPVT-4; Dunn and Dunn 2007). Both are norm-referenced, standardized assessments with adequate reliability and validity that have previously been used successfully with participants with ID (e.g. Loveall and Conners, 2016).

\section{Experimental task and materials}

Participants completed two visual world eye-tracking experiments. In each, participants listened to mini-stories with ambiguous pronouns ( $n=20$ per experiment), such as 'There are the panda and the 


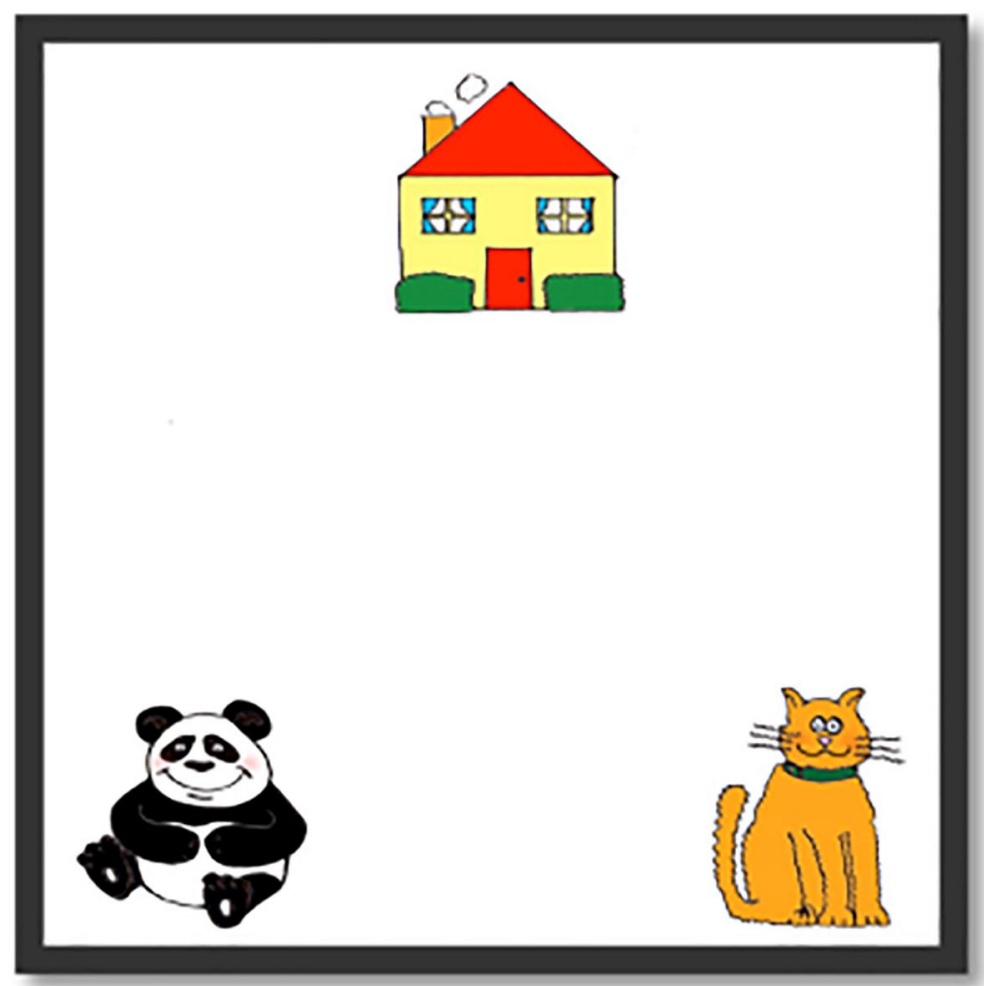

Figure 1. Visual display for the story 'There are the panda and the kitty. The panda tickles the kitty across from the house. He wants to go home'.

kitty. The panda tickles the kitty across from the house. He wants to go home', while looking at corresponding images on the computer screen (Figure 1). All stories followed this grammatical structure and included two animals that served as possible antecedents for the pronoun. Whether a specific animal character (e.g. the panda) was in the subject or object position was counterbalanced across trials to control for the possibility that participants might show a preference for one animal over the other. Order-of-mention was controlled by ensuring that the first-mentioned character in the first sentence was also always the subject and the first-mentioned character of the second sentence. The animate singular male pronoun 'he' was used for all experimental trials.

In Experiment 1, prosodic focus occurred on the subject or object of the sentence preceding the pronoun ( $n=10$ trials each). The focused character was more acoustically prominent: louder, longer and/or produced with slightly higher pitch (e.g. subject focus: 'The PANDA tickles the kitty ...'; object focus: 'The panda tickles 
the KITTY ...'). In Experiment 2, the verb of the sentence preceding the pronoun was either high or low transitivity ( $n=10$ trials each). Transitivity is a semantic property of transitive verbs (those that require both a subject and an object). The subject of a high transitivity verb (e.g. 'kicks') is the agent or do-er of the action, while the subject of a low transitivity verb (e.g. 'smells') experiences the action more passively. The effects of the prosody and transitivity manipulations are not examined in this paper because it is first necessary to determine whether individuals with ID have a subject bias before examining factors that may mitigate such a bias. Therefore, the present study combines data from Experiments 1 and 2 to increase our power to test for the subject bias by using stimuli that contain the types of prosodic and semantic variability that occur in everyday conversation.

\section{Online data}

Participants' eye-gaze to the subject and object characters was monitored using an Eyelink 1000+ eye-tracker. Previous research has established that listeners look more to an image that corresponds to what they are hearing (Tanenhaus et al. 1995), so eye-gaze behavior after pronoun onset is a valid measure of the listener's early, online interpretation of the pronoun (e.g. Järvikivi et al. 2005). Critically, eyetracking does not require a verbal or gross motoric response or metalinguistic decision making, so it offers insight into the participants' processing absent other demands. Eye-tracking has previously been used to test language comprehension in individuals with ID (Brock et al. 2008; Tavares et al. 2015).

\section{Offline data}

Following each story, participants were asked a pronoun interpretation question (e.g. 'Who wants to go home?'). Responses (pointed or verbal) indicated the listener's final, offline interpretation of the pronoun.

\section{Control trials and animal check}

For each experiment, there were 10 additional control trials randomized in with experimental trials. In the control trials, a noun phrase referring to either the subject or object of the previous 
sentence was used in place of the ambiguous pronoun (e.g. '... The mouse nudges the cow next to the leaf. The cow wants ...'). Verbs in control trials were all medium transitivity in order to distinguish them from high and low transitivity verbs used in experimental trials for Experiment 2. Control trials were included to ensure that participants comprehended the stories and were attending to the task. The final sentence referred to the object half of the time, so the control trials also mitigated against the possibility that participants would default to a strategy of assuming that the final sentence refers to the subject of the previous sentence. Before the experiments, participants completed an animal check to ensure familiarity with the animals. If a participant was unable to discriminate between two animals that occurred in the same item, trials involving those animals were excluded from analysis.

\section{Analytic plan}

Data were analyzed with mixed-effects modelling using the lme4 package (Bates et al. 2015) in $R$ (R Core Team 2018). Mixed-effects models can handle unequal sample sizes and missing data. They can also simultaneously account for random effects of participants and items, which were included as random intercepts in all models. All model assumptions were met.

For offline responses, logistic mixed-effects modelling was used because the dependent variable was dichotomous (subject vs. object response). For online responses, linear mixed-effects modelling was used because the data were continuous. The dependent variable was the log transformed proportion of looks to the subject minus object characters; a higher proportion suggests a stronger subject bias. As is common with eye-gaze data (e.g. Arnold et al. 2000; Järvikivi et al. 2005), data were aggregated into discrete time windows for analysis - in this case, 500-ms intervals, from o to 2000 ms after pronoun onset.

First, to examine the subject bias in adults with ID, we tested: (1) if participants showed a subject bias that was significantly different from chance by running null models containing no fixed factors (i.e. independent variables), and (2) if they showed a subject bias that was different from that of TD adults by running models with group as a fixed 
factor. We also ran null models to confirm that participants with TD showed the expected subject bias using our materials. Second, to examine the impacts of non-verbal IQ and vocabulary, we fit models including non-verbal IQ (KBIT-2 non-verbal standard scores) and receptive vocabulary (PPVT-4 growth score values) as fixed factors, which were centered and scaled for analysis.

\section{Results}

We were unable to achieve adequate eye-tracker calibration for three participants with ID in one $(n=1)$ or both $(n=2)$ experiments. Eyegaze data from the remaining participants and partial data for the participant who only completed one experiment are presented in Figure 2. Overall, participants with ID looked more to the subject than the object character, although this was unclear for those with low nonverbal IQs and small vocabularies. Offline, participants with ID selected the subject $57 \%$ of the time; participants with TD selected the subject $92 \%$ of the time.

Results are presented in Table 2. Relative to chance, participants with ID showed a significant or marginal subject bias from o to $500 \mathrm{~ms}, 1000$ to $2000 \mathrm{~ms}$, and offline responses. This subject bias was significantly weaker than that of the TD group at all time windows and in offline responses ( $p$-values $<0.02$ ). The group with TD showed a significant subject bias at all time windows and in offline responses.

For the group with ID, stronger non-verbal abilities were associated with stronger subject biases from o to $1000 \mathrm{~ms}$. The effect of non-verbal IQ was also marginally significant in offline responses, but in the opposite direction: higher non-verbal IQs were associated with fewer subject responses. Larger receptive vocabularies were associated with stronger subject biases from 1000 to $1500 \mathrm{~ms}$ and in offline responses. There were no significant or marginal effects of non-verbal IQ or receptive vocabulary on the subject bias in participants with TD. 

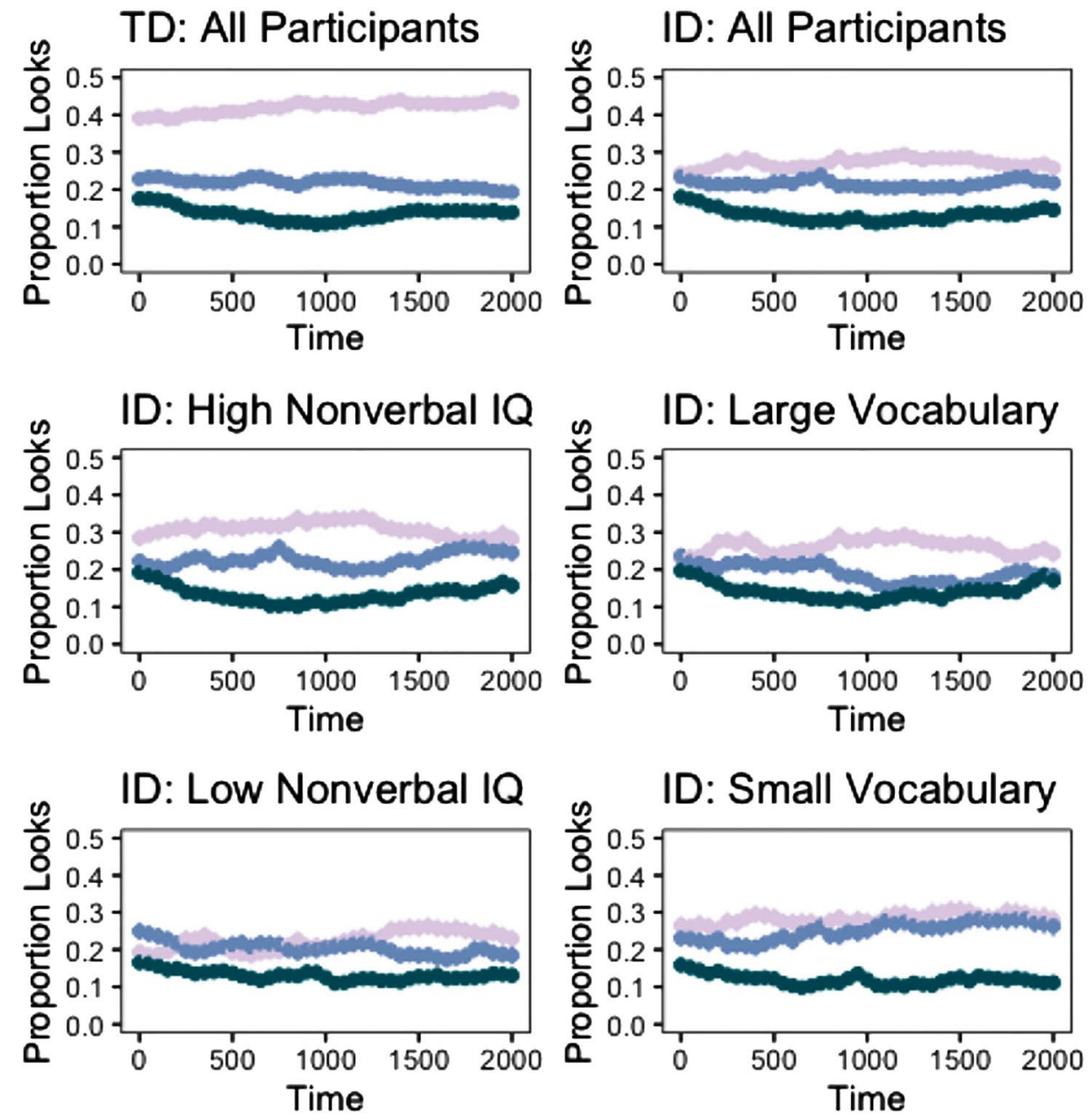

Image $\rightarrow$ Subject $\rightarrow$ Object $\rightarrow$ Location

Figure 2. Proportion looks to the subject, object and location from o to 2000 ms after the onset of the pronoun: the larger the proportion looks to the subject (relative to the object), the stronger the subject bias. For the purposes of this figure, vocabulary and non-verbal IQ were considered high or low based on whether they were above or below the median group score (Table 1).

\section{Discussion}

The first aim of this study was to determine if adults with ID show the subject bias. Results suggest that adults with ID are more likely to interpret an ambiguous pronoun as referring to the subject (vs. object) of the previous sentence. This was evident in both the more-demanding and less-demanding tasks (offline responding to a question 
Table 2 Online and offline results.

\begin{tabular}{|c|c|c|c|c|c|c|c|}
\hline \multirow[b]{2}{*}{ Analysis } & \multirow[b]{2}{*}{ Effect } & \multicolumn{3}{|c|}{$I D$} & \multicolumn{3}{|c|}{$T D$} \\
\hline & & $\beta$ & $S E$ & $P$ & $\beta$ & $S E$ & $P$ \\
\hline \multirow[t]{3}{*}{$\mathrm{o}-500 \mathrm{~ms}$} & Subject bias & 0.36 & 0.16 & $0.028^{*}$ & 1.28 & 0.30 & $<0.001^{*}$ \\
\hline & Non-verbal IQ & 1.18 & 0.43 & $0.006^{*}$ & 0.03 & 0.82 & 0.970 \\
\hline & Vocabulary & -0.06 & 0.21 & 0.783 & -1.96 & 1.77 & 0.246 \\
\hline \multirow[t]{3}{*}{$500-1000 \mathrm{~ms}$} & Subject bias & 0.36 & 0.24 & 0.140 & 1.43 & 0.30 & $<0.001^{*}$ \\
\hline & Non-verbal IQ & 1.30 & 0.64 & $0.038^{*}$ & 0.96 & 0.83 & 0.223 \\
\hline & Vocabulary & 0.12 & 0.31 & 0.688 & -1.51 & 1.80 & 0.377 \\
\hline \multirow[t]{3}{*}{$1000-1500 \mathrm{~ms}$} & Subject bias & 0.56 & 0.24 & $0.025^{*}$ & 1.54 & 0.24 & $<0.001^{*}$ \\
\hline & Non-verbal IQ & 0.61 & 0.59 & 0.257 & 0.14 & 0.63 & 0.807 \\
\hline & Vocabulary & 0.57 & 0.28 & $0.040^{*}$ & -0.90 & 1.36 & 0.486 \\
\hline \multirow[t]{3}{*}{$1500-2000 \mathrm{~ms}$} & Subject bias & 0.33 & 0.17 & $0.057^{\dagger}$ & 1.70 & 0.23 & $<0.001^{*}$ \\
\hline & Non-verbal IQ & -0.35 & 0.49 & 0.443 & 0.01 & 0.65 & 0.991 \\
\hline & Vocabulary & 0.25 & 0.24 & 0.263 & 0.82 & 1.41 & 0.538 \\
\hline \multirow[t]{3}{*}{ Offline responses } & Subject bias & 0.32 & 0.15 & $0.045^{*}$ & 3.11 & 0.34 & $<0.001^{*}$ \\
\hline & Non-verbal IQ & -0.79 & 0.39 & $0.053^{\dagger}$ & 0.74 & 0.81 & 0.359 \\
\hline & Vocabulary & 0.39 & 0.19 & $0.041^{*}$ & 0.54 & 1.69 & 0.749 \\
\hline
\end{tabular}

Results for null models/subject bias are presented in italics, and results for models with KBIT-2 non-verbal IQ and PPVT-4 vocabulary as fixed factors are in regular text.

SE, standard error of the effect estimate ( $\beta$ ); ID, intellectual disability; TD, typical development; KBIT-2,

Kaufman Brief Intelligence Test - 2nd Edition;

PPVT-4, Peabody Picture Vocabulary Test -4 th Edition.

* Statistically significant findings.

† Marginally significant.

and online looking at a computer screen). While this subject bias was significantly greater than chance, it was also weaker than the robust bias seen in typical adult language processing.

In fact, the subject bias displayed by the participants with ID was consistent with that previously reported for 5-year-old TD children in a similar condition of Hartshorne et al. (2015), who reported 65\% offline subject responses (vs. 57\% for our participants). Although the design was different across the two studies, this suggests that the weaker subject bias seen in ID may represent incomplete - rather than deviant - acquisition of this discourse comprehension tool.

The second aim of this study was to examine the relation between the subject bias and non-verbal and verbal abilities in individuals with ID. Our findings suggest that the subject bias may be stronger for individuals with ID who have stronger non-verbal skills at earlier stages 
of processing (o-1000 $\mathrm{ms}$ ) and for individuals with larger vocabularies at later stages (1000-1500 ms) and in offline responses. When offline responses were examined for participants with scores above and below the group's median vocabulary, there were $63 \%$ and 50\% subject responses, respectively, suggesting that the subject bias observed in ID may be driven by those with stronger vocabularies. On the other hand, we saw a marginally weaker offline subject bias for participants with stronger non-verbal skills (53\% vs. 62\% subject responses for those above and below the median non-verbal IQ, respectively). This may be due to participants with high non-verbal IQs preferring to give more varied responses (i.e. avoiding always choosing the subject), because the effect was not seen during online processing. Alternatively, or in addition, it could be partially due to the inability of the KBIT-2 to adequately capture the true variation in our participants' non-verbal abilities ( $n=6$ scored at floor).

This study has important limitations. First, without a matched comparison group of younger TD children, we cannot determine whether the subject bias in ID is in line with verbal or non-verbal ability levels. Future work should examine pronoun processing using such matched groups. Second, while the sample size was adequate to detect significant effects, a larger sample would allow for a more nuanced investigation into the relation between non-verbal and verbal skills and pronoun processing in individuals with ID. Third, it is possible that verbal or non-verbal skills that we did not assess (e.g. general auditory language comprehension, executive function, working memory, general world knowledge and experience) may be more predictive of the subject bias than vocabulary or IQ and therefore would be good avenues for future research. Finally, as discussed in the Introduction, we did not discriminate between the subject bias and the order-ofmention bias. While the two strategies typically lead to the same result in English, this is nonetheless an important distinction. The subject bias reflects linguistic knowledge of who the subject is, while the order-of-mention bias may reflect more a general cognitive primacy effect (see Järvikivi et al. 2005, for discussion). Future work should tease apart these possibilities. 


\section{Conclusions}

The results of this study are an important first step towards understanding if individuals with ID utilize the subject bias (or, perhaps, the order-of-mention bias) when interpreting ambiguous pronouns. Further, our results demonstrate how verbal and non-verbal skills relate to that bias and, given the wide variability in these skills among individuals with ID, help us understand the generalizability of our results to the larger population. Successful communication requires a listener to determine which potential referent a talker intends to refer to each time she or he uses a pronoun. Errors in pronoun resolution can significantly derail comprehension - not only of an individual sentence but also of the broader conversation. Individuals with ID do appear to use the subject bias, although the effect is weaker than that seen in adults with TD. Explicitly teaching and strengthening the subject bias alongside other pronoun interpretation strategies, as well as developing verbal and/or non-verbal skills that could promote pronoun interpretation, may help individuals with ID improve their language comprehension abilities. In addition, previous work has also found that explicit instruction in identifying the referent of a pronoun is helpful in fostering reading comprehension (Dommes et al. 1984).

Acknowledgments Earlier versions of this work were presented at the American Speech-Language Hearing Association Convention in 2018 and at the 51st Annual Gatlinburg Conference. Thank you to Juhani Järvikivi and Anja Arnhold, as well as Beka Bosley, Sarah Fischer, Kimberly Gibson, Alix Moody, Madison Savoy, Andie Zang and other students involved in data collection and entry. We are deeply grateful to North Mississippi Regional Center and all the individuals who participated.

Source of funding Research funds from the University of Mississippi were used to support this project.

Conflict of Interest The authors certify that there are no conflicts of interest.

Data Availability Statement The data that support the findings of this study are available from the corresponding author upon reasonable request. 


\section{References}

Arnold J. E., Eisenbrand J. G., Brownd-Schmidt S. \& Trueswell J. C. (2000) The rapid use of gender information: evidence of the time course for pronoun resolution from eye-tracking. Cognition 76, 13-26.

Bates C., Maechler M., Bolker B. \& Walker S. (2015) Fitting linear mixed-effects models using lme4. Journal of Statistical Software 67, 1-48.

Brock J., Norbury C., Einav S. \& Nation K. (2008) Do individuals with autism process words in context? Evidence from language-mediated eye-movements. Cognition 108, 896-904.

Dommes P., Gersten R. \& Carnine D. (1984) Instructional procedures for increasing skill-deficient fourth graders' comprehension of syntactic structures. Educational Psychology 4, 155-65.

Dunn L. M. \& Dunn D. M. (2007) Peabody Picture Vocabulary Test-Fourth Edition. American Guidance Service, Circle Pines, MN.

Frederiksen J. (1981) Understanding anaphora: rules used by readers in assigning pronominal referents. Discourse Processes 4, 323-47.

Gernsbacher M. A. \& Hargreaves D. J. (1998) Assessing sentence participants: the advantage of first mention. Journal of Memory and Language 27, 699-717.

Hartshorne J. K., Nappa R. \& Snedeker J. (2015). Development of the first-mention bias. Journal of Child Language 42, 423-46.

Hawthorne K., Arnhold A., Sullivan E. \& Järvikivi J. (2016). Social cues modulate cognitive status of discourse references. Proceedings of the 38 th Annual Meeting of the Cognitive Science Society, 562-567.

Järvikivi J., van Gompel R. P. G., Hyönä J. \& Bertram R. (2005) Ambiguous pronoun resolution: Contrastive the first-mention and subject preference accounts. Psychological Science 16, 260-4.

Kaufman A. S. \& Kaufman N. L. (2004) Kaufman Brief Intelligence Test, Second Edition Manual. AGS, Circle Pines, MN.

Loveall S. J. \& Conners F. A. (2016) Reading skills in Down syndrome: an examination of orthographic knowledge. American Journal on Intellectual and Developmental Disabilities 121, 95-110.

Perovic A. (2006) Syntactic deficit in Down syndrome: more evidence for the modular organization of language. Lingua 116, 1616-30.

Perovic A., Modyanova N. \& Wexler K. (2013) Comprehension of reflexive and personal pronouns in children with autism: a syntactic or pragmatic deficit. Applied PsychoLinguistics 34, 813-35.

R Core Team (2018) R: A Language and Environment for Statistical Computing. R Foundation for Statistical Computing, Vienna, Austria. Available at: https:// www.Rproject.org/

Song H. F. \& Fischer C. (2005) Who's "she”? Discourse prominence influences preschoolers' comprehension of pronouns. Journal of Memory and Language $52,29-58$. 
Song H. J. \& Fisher C. (2007) Discourse prominence effects on 2.5-year-old children's interpretation of pronouns. Lingua 117, 1959-87.

Tanenhaus M. K., Spivey-Knowlton M. J., Eberhard K. M. \& Sedivy J. C. (1995) Integration of visual and linguistic information in spoken language comprehension. Science 268, 1632-4.

Tavares G., Fajardo I., Avila V., Salmeron L. \& Ferrer A. (2015) Who do you refer to? How young students with mild intellectual disability confront anaphoric ambiguities in texts and sentences. Research in Developmental Disabilities 38, 108-24.

Tyler L. K. \& Marslen-Wilson W. (1982) The resolution of discourse anaphors: Some online studies. Text-Interdisciplinary Journal for the Study of Discourse 2, 263-91. 